\title{
A Robust Neural Network Algorithm For Automotive Air Conditioning Fault Detection
}

\author{
Dinh Anh Tuan Tran'), Huong Nguyen Thi Cam²), Quoc Minh Phan ${ }^{1)}$ \\ 1) Faculty of Heat \& Refrigeration Engineering, Industrial University of Ho Chi Minh City, Ho Chi Minh 700000, Viet Nam (E-mail: \\ trandinhanhtuan@iuh.edu.vn)
}

\author{
2) FPT University, Ho Chi Minh 700000, Viet Nam.
}

Received on October 12, 2019

\begin{abstract}
Accurate and incipient fault detection of air conditioning systems is highly demanded in a car to prevent energy waste and high maintenance cost. However, most fault detection techniques require experiences of drivers which are usually unavailable. In this study, a novel hybrid method is proposed to detect faults for AC systems in car. Two typical faults in AC system are adopted to investigate. An AC fault detection and diagnosis framework is introduced by combining the RBFNN model and the EWMA. The results show that the proposing algorithm detects typical air conditioning faults in a car with high accuracy.
\end{abstract}

KEYWORDS: Radial basis function neural network, Exponentially-weighted moving average, Fault detection and diagnosis, Automotive air conditioning (A1)

\section{Introduction}

Nowadays, it is undeniable that the cars have become one of integral means for human condition. The demand for car appears to be on rise while the gasoline is limited in the world. Therefore, the energy saving in vehicle has recently become a topical approaches in a number of previous researches ${ }^{[1][2][3]}$. Recently, scientists at the National Academics of Science in USA were figured out that vehicle consumes enormous amount of energy, i.e. up to $58 \%$ of the total fuel. Meanwhile, there are numerous factors such as mechanical failure, skill driver, $\mathrm{AC}$ system ... that can lead to an increase in fuel consumption of a car. Among these accessories, the AC unit, which is one of indispensable components due to its thermal comfort in the modern car, requires far more energy than any others. It need up to $30 \%$ of the fuel consumption ${ }^{[3]}$, even more 27 billion liters of gasoline every year in $\mathrm{US}^{[2]}$. Therefore, the decrease of the energy consumption in the air-conditioning system has become a tense concern for the automotive industry. Generally, the energy wastage was directly under the influences of failure performance in $\mathrm{AC}$ unit. According to published articles ${ }^{[4][5]}$, around $20-30 \%$ waste of energy consumption was estimated by faults and non-optimal operations in air conditioning system. In the meantime, it was also reported that approximately $10-30 \%$ of energy consumption will be saved if faults detection is incipiently implemented in $\mathrm{AC}$ system ${ }^{[6]}$. Consequently, to obtain this purpose, the development of a capable fault detection (FDD) strategy is significantly necessary for maintaining the AC system in normal steady condition as well as operating cost saving.

Recently, a range of scholars on fault detection and diagnosis for AC unit have been conducted. In general, there are relatively two categories of fault detection and diagnosis: model-based and model-free based FDD method. Of those, the accuracy of the model-based FDD method have somewhat more dominant than model-free based FDD method. The main reason relies on the model-free FDD methods usually employ straightforwardly structures to capture the complex unit operations as the system
AC system. Otherwise, the model-based FDD methods widely exert a mathematical model to analyze relations between the measured variables such as Regression model, Principal component analysis (PCA), Least squares support vector regression (LSSVR), Kriging and Artificial neural networks. Particularly, the Regression model was carried out by Fu et al. ${ }^{[7]}$ to predict the health of chiller system. The PCA method was developed by Fu et al. ${ }^{[8]}$, Guo et al. ${ }^{[9]}$ to detect and diagnose faults in VRF system which is a kind of air-conditioning system. Similarly, the implementation of fault detection and diagnosis is also proposed by a range of methods such as the Support vector regression ${ }^{[10][5][11]}$, the Kriging method ${ }^{[12]}$. The Artificial neural networks (ANNs) have also adopted in various research fields over the recent years ${ }^{[13][14][15]}$. Related to application of ANNs, researchers have widely approved that the Artificial neural networks have powerful abilities over traditional statistic fields for improving accuracy rate of fault detection and diagnosis results. Therefore, this study establishes a fault diagnosis model based on a novel radial basis function (RBF) neural network which is a sort of the ANNs and investigates its potential in air conditioning system on car to enhance energy saving purposebuilt.

\section{Methodology and experimental data}

2.1. Development of RBFNN reference model

In this study, the radial basis function (RBF) neural networkbased method is proposed as a reference model. The RBFNN is an effective feed-forward neural network. Over decades, it has been widely employed in considerable fields such as approximation, classification, regression problems, prediction, signal processing, and other problems due to its good performance in approximation and global optimum. The RBFNN structure has three layers including input layer, hidden layer, and output layer (see Fig. 1). 
For the input layer, the data of input vectors should be preprocessed such as normalization processing technique before the input vectors are input to the RBFNN network.

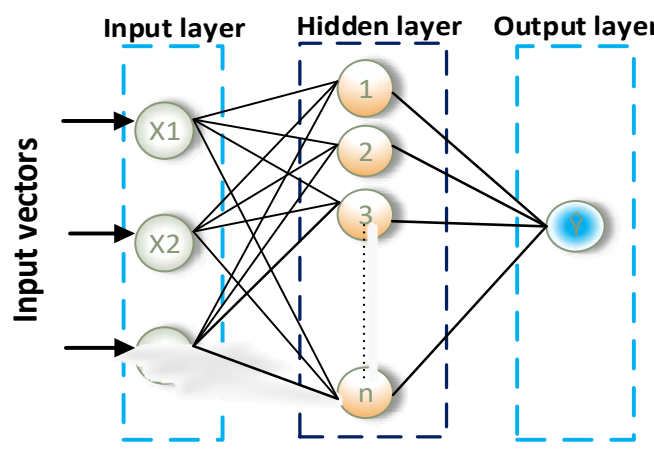

ig. 1 The basic structu re of RBF neural netwo rk

The hidden layer (RBF layer) is composed of hidden neurons. Each hidden neuron has a radial basis function which is a center symmetric nonlinear function based on Euclidean distance denotes as $\|$.$\| . The basis function play activation role and can$ take different types of interpolating function such as Multiquadric Inverse multiquadric, Inverse quadratic, Gaussian. Among them, the Gaussian of RBF is popularly employed as the radial basis function, that is shown below:

$$
\emptyset_{i}(x)=\exp \left[\frac{\left\|x-\mu_{i}\right\|^{2}}{2 \sigma_{i}^{2}}\right]
$$

where $\mu_{i}$ and $\sigma_{i}$ are denoted as the center and spread width of the $i_{\text {th }}$ node.

The output layer of $m$ outputs is a vector $\mathrm{y}=\left[\mathrm{y}_{1}, \mathrm{y}_{2} \ldots \mathrm{y}_{m}\right]$ which is gained by the linear combination of the hidden nodes. Its output can be presented as below:

$$
y=\widetilde{f}(x)=\sum_{i=1}^{n} \omega_{i} \emptyset_{i}(x)
$$

where $\omega_{\mathrm{i}}$ is the unknown weighting coefficient to be determined by the least-square with supervised learning method.

\subsection{Faults selection and simulated experimental faults}

As aforementioned, the fault detection of $\mathrm{AC}$ system on the modern vehicles plays a crucial role in maintaining well-being operating as well as saving energy. Typically, there are a range of faults in AC system. However, it can be roughly divided into two major parts: abrupt and gradual fault. In fact, in the case of the abrupt faults such as locked compressor, burned magnetic compressor coil, broken pipe are easily addressed due to theirs clear symptoms. Therefore, they are not considerable objects in this study. Otherwise, gradual faults including refrigerant overcharge, refrigerant leakage, non-condensable gas, evaporator air blockage, condenser air blockage may take place slowly. They are still not easy to detect. Usually, they can be found when their damage indicators are seriously. It means that the system is facing various issues of operation. Among these faults, the refrigerant leakage and the condenser air blockage are chosen due to frequent appearing under Vietnam conditions.

\subsubsection{Experimental data and method}

In this study, a conventional AC system including a beltdriven compressor, an air cooled condenser, a laminar microchannel evaporator, a thermostatic expansion valve (TXV) was installed to simulate experiments in the development and evaluation of proposed fault detection and diagnostic (FDD) methods. The simulated faults and the fault conditions are given in the Table 1 .

Table 1 Methods of implementing the faults and levels simulated

\begin{tabular}{|l|c|c|c|c|c|l|}
\hline \multirow{2}{*}{ Faults } & \multicolumn{5}{|c|}{ Severity levels of faults } & Methods of simulated \\
\cline { 2 - 6 } & $\begin{array}{c}\text { Fault } \\
\text { free }\end{array}$ & $\begin{array}{c}\text { Level } \\
1\end{array}$ & $\begin{array}{c}\text { Level } \\
\text { faults }\end{array}$ & $\begin{array}{c}\text { Level } \\
3\end{array}$ & $\begin{array}{c}\text { Level } \\
4\end{array}$ & $\begin{array}{l}\% \text { reduction of the total } \\
\text { amount charge }\end{array}$ \\
\hline $\begin{array}{l}\text { Refrigerant } \\
\text { leakage }\end{array}$ & $0 \%$ & $\begin{array}{c}- \\
5 \%\end{array}$ & $-10 \%$ & $-20 \%$ & $-30 \%$ & \\
\hline $\begin{array}{l}\text { Condenser air } \\
\text { blockage }\end{array}$ & $0 \%$ & $-10 \%$ & $-20 \%$ & $-30 \%$ & $-40 \%$ & $\begin{array}{l}\text { block the condenser coil } \\
\text { with volume control } \\
\text { damper }\end{array}$ \\
\hline
\end{tabular}

The fault free and faults data including both transient and steady state conditions were collected by PNTECH CONTROLS DDC-C46 and relayed to the PC through Modbus RTU 485 with 3 -second sampling intervals. To enhance the accuracy of a model, the quality of data plays a vital role in training and validating model. Therefore, these data will go through a data pre-processor which is formed by a measurement moving-window involving three standard deviations from mean and 10 testing points to remove messy data, firstly. Remaining data will be divided into different data sets as training, validation and testing dataset in this study.

\subsubsection{Basic structure of the FDD strategy}

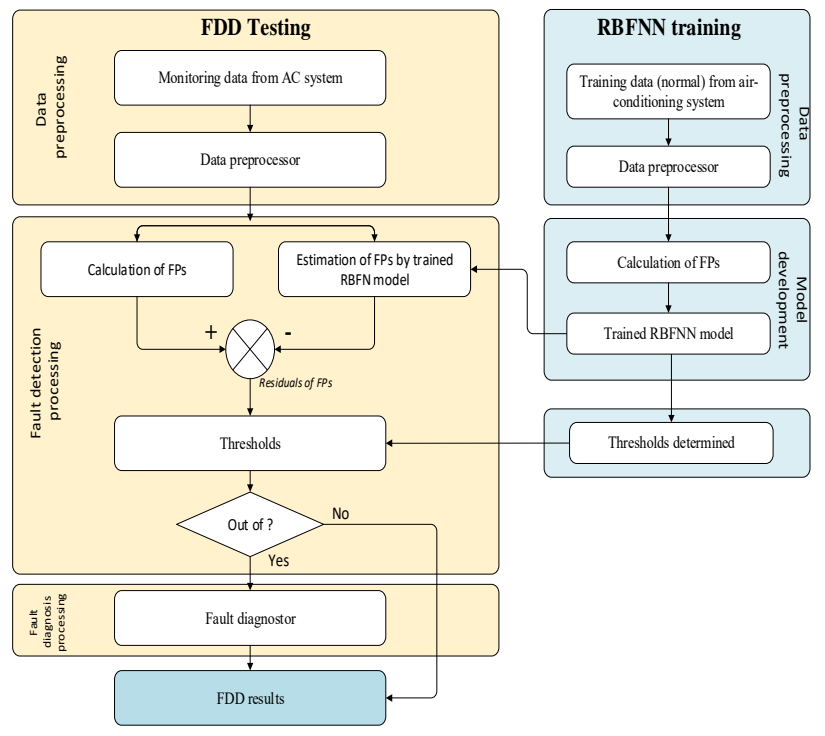

Fig.2 The basic implementation structure of proposed FDD strategy

The basic structure of the proposed FDD strategy is given in Fig.2. It has two main components, i.e. the RBFNN models training and the FDD testing. The reference models training contains four steps: data pre-processing, feature parameter (FP) calculation, reference model development and calculation of limited checking thresholds. The selection of feature parameters related to faults play a crucial role in the reliability and sensitivity of a FDD strategy in a AC system. They have physically meaningful and sensitive to the concerned faults to capture the health state of the AC system. Therefore, the feature parameters, $\mathrm{T}_{c d}$ (condenser temperature) and $\mathrm{T}_{s c}$ (sub-cooling temperature) 
which are sensitive to condenser fouling and refrigerant leakage are employed as a output of RBFNN reference models in this study. The reference models RBFNN of two feature parameters with the form of $\mathrm{Yi}=\mathrm{f}\left(\mathrm{Q}_{\mathrm{ev}}, \mathrm{T}_{c e}, \mathrm{~T}_{c o}\right)$ have basically three independent variables, i.e., the cooling load $\left(\mathrm{Q}_{\mathrm{ev}}\right)$, the entering condenser air temperature $\left(\mathrm{T}_{c e}\right)$ and the leaving condenser air temperature $\left(\mathrm{T}_{c o}\right)$ to calculate the benchmarks of feature parameters in normal condition. The constant air flow in condenser is assumed for stable during test.

In the test of trained RBFNN model part, the steps of data preprocessing and FPs calculation are as similar as that in the reference models training part. The benchmarks of the feature parameters are also created by their corresponding reference models. Then, the residuals which are difference between the current FPs and benchmark values, are inputs of the Exponentially Weighted Moving Average (EWMA) control charts to determine limit checking. The EWMA is a powerful tool of statistical quality control in detection small shift of a process. Since its advantages have proven in a number of previous researches ${ }^{[5][11]}$, the EWMA is still adopted in this study. The limit checking including upper and lower control limits of each feature parameter is computed by:

$$
\begin{aligned}
& U C L=\mu_{0}+L \cdot \sigma \sqrt{\frac{\lambda}{n(2-\lambda)}} \\
& L C L=\mu_{0}-L \cdot \sigma \sqrt{\frac{\lambda}{n(2-\lambda)}}
\end{aligned}
$$

where $\mathrm{L}$ is the width of the control limits with a confidence level $\alpha$. The selection of the width value for control limits is a crucial aspect to FDD strategy. It is a compromise between the sensitivity of the FDD process and the likelihood of sounding a false alarm. Increasing the threshold will reduce false alarms but reduce the sensitivity of the FDD process, and vice versa. In this study, the width of the control limits $\mathrm{L}$ is set as 3 (i.e. the confidence level is $99.73 \%) . \sigma$ is the standard deviation of the residuals. $\mu_{0}$ is the expectation of the residuals. The target or center line for the control chart is the average of historical residual data.

If the residual is within the width of control limits chart, the system has no fault. Otherwise, a fault is detected, it is further identified by using the fault diagnosis rule-based in the below table 2. The rule-set in Table 2 are relied on the impacts of the faults on the feature parameters. Particularly, condenser fouling is a kind of component physical fault. The rate of heat transfer is directly proportional to relevant surface area of a condenser. When condenser fouling occurs, the heat transfer surface area for condensing process will be reduced. Consequently, this fault results in higher $\mathrm{T}_{c d}$. For the refrigerant leakage, the lack of refrigerant mount in the $\mathrm{AC}$ system will naturally cause the condenser pressures and condensing temperatures to be lower, which leads to smaller $\mathrm{T}_{s c}$.

Table 2 Faults diagnosis rule-set

\begin{tabular}{|l|c|c|}
\hline \multirow{2}{*}{ Fault types } & \multicolumn{2}{|c|}{ Symptom of feature parameters } \\
\cline { 2 - 3 } & $\mathrm{T}_{c d}$ & $\mathrm{~T}_{s c}$ \\
\hline Condenser fouling & $\uparrow$ & - \\
\hline Refrigerant leakage & - & $\downarrow$ \\
\hline
\end{tabular}

Where the symbol “个” or " $\downarrow$ " is an increased or decreased trend of feature parameter under impact of fault. The symbol, "-“, has no interaction under fault.

\subsubsection{Evaluation method}

The evaluation of a reference model is an one of necessary task in FDD strategy to measure the success of training model performance. For this reason, the values of two popularly different accuracy measures are used, i.e. the R-square $\left(R^{2}\right)$ and the mean absolute error (MAE) which are calculated by Eqs. (5) and (6), respectively as below:

$$
\begin{aligned}
& \mathrm{R}^{2}=1-\sum_{\mathrm{i}=1}^{\mathrm{n}}\left(\mathrm{y}_{\mathrm{i}}-\hat{\mathrm{y}}_{\mathrm{i}}\right)^{2} / \sum_{\mathrm{i}=1}^{\mathrm{n}}\left(\mathrm{y}_{\mathrm{i}}-\overline{\mathrm{y}}_{\mathrm{i}}\right)^{2} \\
& \mathrm{MAE}=\frac{\sum_{\mathrm{i}=1}^{\mathrm{n}}\left|\mathrm{y}_{\mathrm{i}}-\widehat{\mathrm{y}}_{\mathrm{i}}\right|}{\mathrm{n}}
\end{aligned}
$$

where $y_{i}$ and $\hat{y}_{i}$ are the calculated/measured and predicted values of the feature parameter ith, $\sigma$ is the standard deviation, and $\mathrm{n}$ is the number of sample points. The accuracy assessment of a model is a compromise of both measures. The larger the value of Rsquare and the smaller the value of MAE, the more accurate the model is.

\section{FDD test results and discussion}

\subsection{Evaluation of reference model}

In this study, the remained training data set which has approximately 136 data points is employed to evaluate the accuracy of reference model. Based on the Eqs.(5\&6), the values of the two measurement indicators are 0.99308 of R-square, 0.000054 of MAE for $\mathrm{T}_{c d}$ and 0.99964 of R-square, 0.000565 of MAE for $\mathrm{T}_{s c}$, respectively.

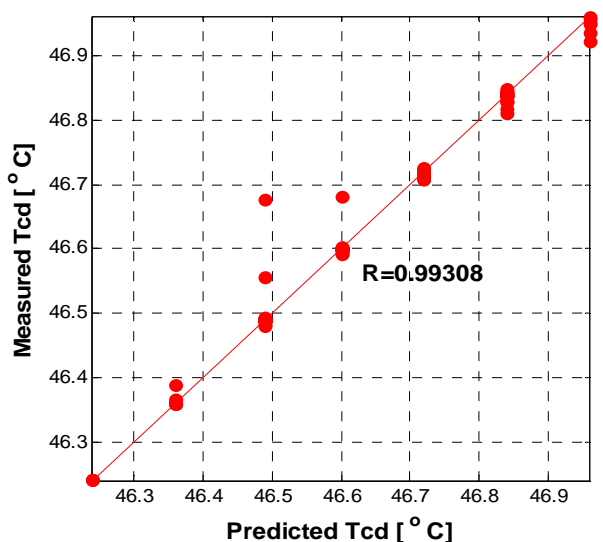

(a)

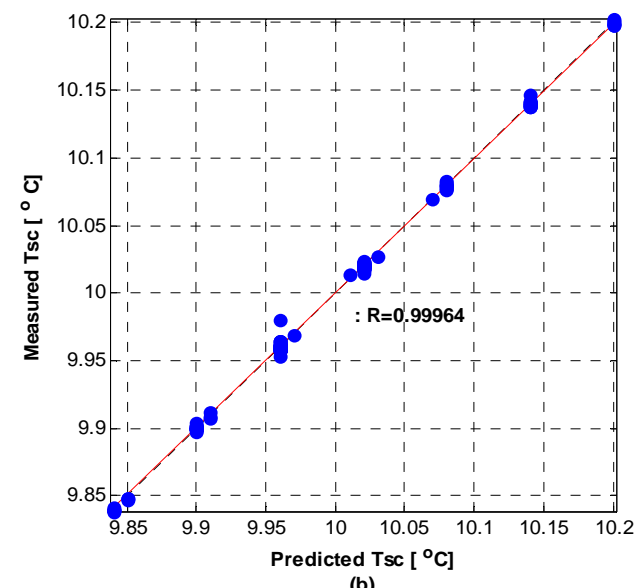

(b) 
Vol.11, No.1(2020)

Fig.3 Comparisons between predicted and measured values of feature parameters using training data from "normal" dataset.

The Figure 3 illustrates the comparisons between predicted and measured feature parameters. It can be obviously found that both coefficient of determinations $\left(\mathrm{R}^{2}\right)$ are quite high, the MAEs are small that show the strong goodness-of-fit of RBFNN models.

\subsection{The results of FDD performance}

To determine the accuracy of FDD performance, the correct ratio which equals the amount of correct sample points to total amount of sample points is used in this study. As aforementioned, the correct sample points mean that they satisfy the rule-based diagnosis. Particularly, for the fault-free test case, the correct sample points should be lied within limit checking boundaries. For the condenser fouling, the correct sample points of $\mathrm{T}_{c d}$ is higher than upper control limit. Similarly, the correct sample points of $\mathrm{T}_{s c}$ is lower than lower control limit.

The FDD test results of normal and faults case are illustrated in the Figure 4. In this figure, the red lines are the results of upper and lower control limit which are calculated by Eqs. (3) \& (4) at the $99.73 \%$ confidence level. The $\mathrm{x}$-axis corresponds to the samples of normal data and the increasing order of the four severity levels data from left to right. The y-axis corresponds to the residuals of each feature parameter gaining from trained RBFNN models. The residuals of two feature parameters, i.e. $\mathrm{T}_{c d}$ and $\mathrm{T}_{s c}$, are depicted in blue color for normal (fault-free) case, in red color for condenser fouling case, in green for refrigerant leakage case.
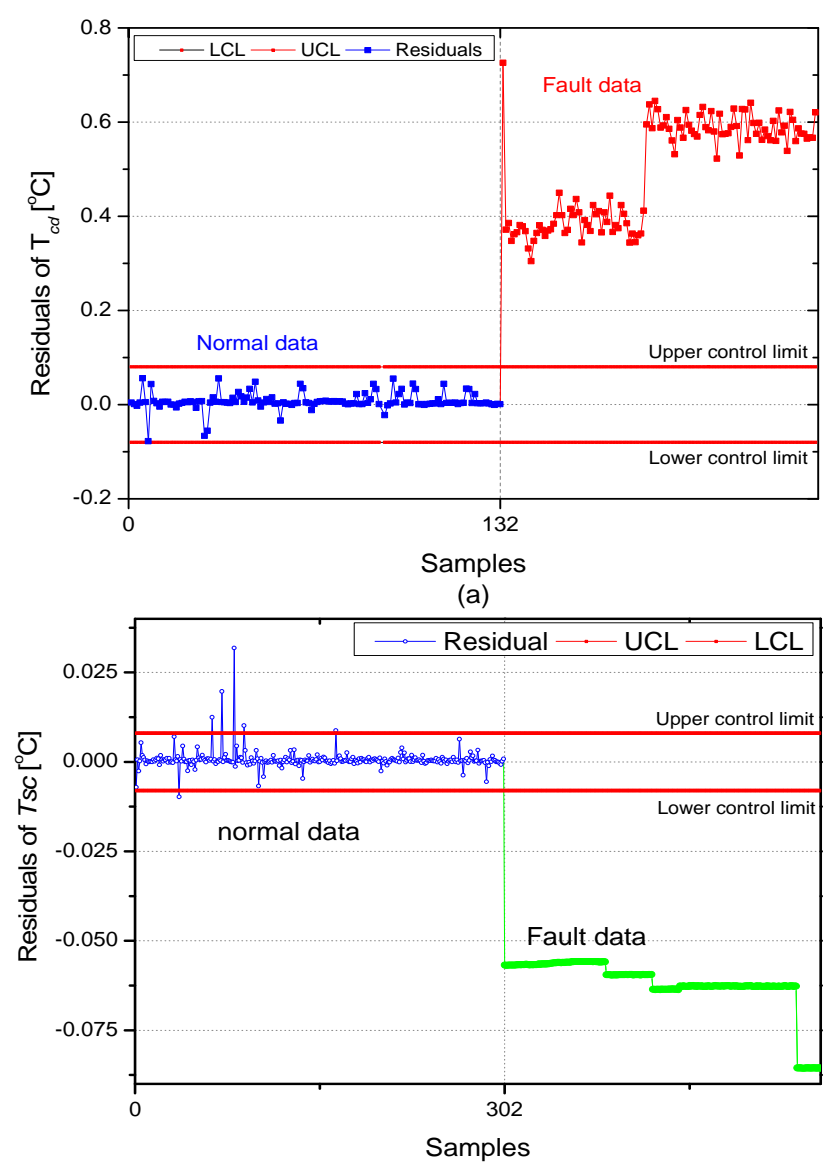

(b)
Fig. 4 The results of validation to proposed method: (a) The sympton of $\mathrm{T}_{c d}$ residual under condenser fouling;(b) The sympton of $\mathrm{T}_{s c}$ residual under refrigerant leakage

From the illustration of the Figure 4, it is easily found that all residuals of $\mathrm{T}_{c d}$ and almost residuals of $\mathrm{T}_{s c}$ are in checking limit. There are only few residuals of $\mathrm{T}_{c d}$ are out of checking limit. Therefore, the rate of correct diagnosis are $100 \%$ for $\mathrm{T}_{c d}, 98 \%$ for $\mathrm{T}_{s c}$. Although it still has $2 \%$ of false diagnosis rate for $\mathrm{T}_{s c}$, it can be found that the implementation of the proposed method clearly has significant contributions in the enhancement of FDD. For two faults test, all residuals of both $\mathrm{T}_{c d}$ and $\mathrm{T}_{s c}$ completely exceed the checking limit. Particularly, all residuals of $\mathrm{T}_{c d}$ are higher than upper control limit and all residuals of $\mathrm{T}_{s c}$ are lower than lower control limit. These results show that the diagnosis performances of proposed method gain $100 \%$ of correct diagnosis rate and their symptom correspond to diagnosis rule-based of proposed method. Therefore, it could be recognized the proposed method has enough ability to detect and diagnose the suggested faults of AC system on a car.

\section{Conclusion}

In this paper, a robust and effective FDD strategy for the AC system in a car is developed. We employed the EWMA control charts to determine checking limit for development of fault detection. Two well-known measurement indicators such as Rsquare and the mean absolute error (MAE) are approved to evaluate the accuracy of a reference model performance. The values of these two measurement indicators show that the accuracies of RBFNN model can serve as a predictable reference model in FDD strategy. RBFNN is adopted as a reference model to capture the behaviors of $\mathrm{AC}$ system in a car. The reference models provided clear information of $\mathrm{AC}$ system status. Both fault free and two typical kinds of $\mathrm{AC}$ faults data were used to implement the FDD strategy. Results of AC fault detection and diagnosis performance proved that proposed method has the capability to detect and diagnose typical AC system faults.

\section{References}

[1] B. Mebarki, B. Draoui, B. Allaou, L. Rahmani, E. Benachour, Impact of the air-conditioning system on the power consumption of an electric vehicle powered by lithiumion battery, Model. Simul. Eng. 2013 (2013).

[2] K. Shete, Influence of Automotive Air Conditioning load on Fuel Economy of IC Engine Vehicles, Int. J. Sci. Eng. Res. 6 (2015) 1367-1372.

[3] A. Subiantoro, K.T. Ooi, U. Stimming, Energy Saving Measures for Automotive Air Conditioning ( AC ) System in the Tropics, 15th Int. Refrig. Air Cond. Conf. (2014) 1-8.

[4] Z. Ma, S. Wang, Building energy research in Hong Kong: A review, Renew. Sustain. Energy Rev. 13 (2009) 1870-1883.

[5] Y. Zhao et al., A statistical fault detection and diagnosis method for centrifugal chillers based on exponentially weighted moving average control charts and support vector regression, Appl. Therm. Eng. 51 (2013) 560-572.

[6] H. Wang, Y. Chen, C.W.H. Chan, J. Qin, An online fault 


\section{Dinh Anh Tuan Tran et al / International Journal of Automotive Engineering}

Vol.11, No.1(2020)

diagnosis tool of VAV terminals for building management and control systems, Autom. Constr. 22 (2012) 203-211.

[7] F. Xiao, C. Zheng, S. Wang, A fault detection and diagnosis strategy with enhanced sensitivity for centrifugal chillers, Appl. Therm. Eng. 31 (2011) 3963-3970.

[8] F. Xiao, S. Wang, X. Xu, G. Ge, An isolation enhanced PCA method with expert-based multivariate decoupling for sensor FDD in air-conditioning systems, Appl. Therm. Eng. 29 (2009) 712-722.

[9] Y. Guo, G. Li, H. Chen, Y. Hu, H. Li, J. Liu, M. Hu, W. Hu, Modularized PCA method combined with expert-based multivariate decoupling for FDD in VRF systems including indoor unit faults, Appl. Therm. Eng. 115 (2017) 744-755.

[10] J. Liang, R. Du, Model-based Fault Detection and Diagnosis of HVAC systems using Support Vector Machine method, Int. J. Refrig(2007)1104-1114.

[11] Y Chen, D. Anh, T. Tran, L. Ao, N. Thi, C. Huong, An enhanced chiller FDD strategy based on the combination of the LSSVR-DE model and EWMA control charts, Int. J. Refrig. (2016).

[12]Y M. Chen, C.-L. Jiang, D.A.T. Tran, A fault detection and diagnosis method based on kriging model for chillers, Hunan Daxue Xuebao/Journal Hunan Univ. Nat. Sci. (2016).

[13]W. Wang, Z. Xu, J. Weizhen Lu, Three improved neural network models for air quality forecasting, Eng. Comput. 20 (2003) 192-210.

[14]M. Awad, H. Pomares, I. Rojas, O. Salameh, M. Hamdon, Prediction of Time Series Using RBF Neural Networks: A New Approach of Clustering, 6 (2009) 138-144.

[15]Y. Ze, F. Cell, Federal Funds Rate Prediction : A Comparison Between the Robust RBF Neural Network and Economic Models, Journal of Information Science and Engineering, 25(3), (2009) 763-778.

[16]J. Cui, S. Wang, A model-based online fault detection and diagnosis strategy for centrifugal chiller systems, Int. J. Therm. 